\title{
ASSESSING THE POTENTIAL OF MEDICAL-MASK AND DRUG-TREATMENT IN CONTROLLING INFLUENZA DISEASE
}

\author{
M. Rohmah ${ }^{1}$, B.D. Handari ${ }^{2}$, D. Aldila ${ }^{3}$ \\ 1,2,3 Department of Mathematics \\ Universitas Indonesia \\ Kampus UI Depok \\ Depok 16424, INDONESIA
}

\begin{abstract}
A classical SIS model for influenza disease with two intervention strategies such as medical mask and drug treatment from deterministic framework was extended in this paper into a stochastic differential equation (SDE). The SDE model was constructed by introducing a random perturbation in successful contact rate parameter. The concern of this study is focused in to two aspects based on from mathematical and epidemiological point of view. From mathematical point of view, the basic reproduction number from deterministic framework (known as $R_{0}$ ) was analyzed and compared with the stochastic threshold parameter (we call it as $\rho_{0}$ ). As well as $R_{0}$ in deterministic model, the $\rho_{0}$ also linked into the extinction and persistence condition of endemic equilibrium. If $\rho_{0}<1$, then the SDE system will reach the extinction of influenza disease with probability one. Otherwise, if $\rho_{0}>1$ then influenza disease will persist. However, we found a situation where $\rho_{0}$ gives a contrast result compared to $R_{0}$ as a consequence of random perturbation in successful contact rate parameter. From epidemiological point of view, we found that medical mask and drug treatment intervention and successful contact rate influence the dynamic of influenza spread in our model, which can give us a direction about the best strategy to regulate influenza spreads. Some numerical simulations were generated to support the analytical results.
\end{abstract}

Received: January 14, 2017

Revised: $\quad$ April 26, 2017

Published: $\quad$ August 7, 2017

${ }^{\S}$ Correspondence author (c) 2017 Academic Publications, Ltd. url: www.acadpubl.eu 
AMS Subject Classification: 92B05

Key Words: influenza, deterministic, stochastic differential equations, medical mask, drug treatment, basic reproductive ratio, stochastic threshold parameter

\section{Introduction}

Influenza, also called as Flu, is a respiratory illness caused by influenza viruses. People infected by influenza virus show some symptoms like fever, headaches, sore throat or cough. This disease is easily spread among human, mainly from droplets made when infected people talk, sneeze or cough $[4,10]$. The most important thing to prevent people from infection of influenza is by taking a vaccination each year. Also it is recommended to staying away from infected people or prevent yourself by wearing a medical mask (it is more recommended that infected person is the one who should wear medical mask [6]).

Mathematical modelling can lead the way in purpose of understanding the complexity of various disease and could become as the scientific back-up for controlling the disease before implementation in to the field. Since Kermack and Mckendric [7] introduced the SIR model to understand the dynamic of epidemic system, many mathematical models have been proposed since then with the intention to understand the qualitative behaviour for various disease like dengue $[15,16,18]$, malaria $[11,12]$, West Nile virus $[13,14]$, Influenza [6, 19], swine flu [17], etc. Many mathematical model have been developed to understand the spread of influenza and explored strategies for control the spread $[6,19,20,21,22]$. In this article, we will reconstruct the influenza model in [6] as a Stochastic Differential Equation (SDE) model. The model in [6] was introduced as a two dimensional deterministic model with involving intervention of medical mask and drug treatment interventions, and given by :

$$
\left.\begin{array}{l}
\frac{d \bar{S}}{d t}=-\frac{\left(p \beta u_{1} \bar{S}+\left(1-u_{1}\right) \beta \bar{S}\right) \bar{I}}{N}+\left(\alpha+\left(\gamma_{0}+\gamma_{1} u_{2}\right)\right) \bar{I} \\
\frac{d \bar{I}}{d t}=\frac{\left(p \beta u_{1} \bar{S}+\left(1-u_{1}\right) \beta \bar{S}\right) \bar{I}}{N}-\left(\alpha+\left(\gamma_{0}+\gamma_{1} u_{2}\right)\right) \bar{I}
\end{array}\right\} .
$$

with $\bar{S}$ and $\bar{I}$ represent the number of susceptible and infected person, $u_{1}, u_{2} \in$ $[0,1]$ as medical mask and drug treatment intervention respectively, $\alpha>0$ as natural death rate, $\gamma_{0}, \gamma_{1} \geq 0$ as the natural and drug-induced recovery rates respectively, $\beta>0$ as successful infection rate and $p \in[0,1]$ as a reduction of $\beta$ because of medical mask use. The $\beta$ parameters in the deterministic model is a constant all the time, while in the real world, $\beta$ is not constant all the time. This fact will be discussed in the model that we proposed in this article. 
In the next section, the proposed method will be discussed and followed with some results in the third section. Some conclusions will be given in the last section.

\section{The Proposed Method}

In this section, the development of the model in 1 will be given as an SDE model in first sub-section and then followed with statistical analysis about the persistence and coexistence of equilibrium points in second sub-section.

\subsection{The SDE Model}

Given the deterministic model for influenza spread with medical mask $\left(u_{1}\right)$ and treatment $\left(u_{2}\right)$ in [6]. The model assumed constant parameters, whereas in reality, the nature of epidemic growth and spread is inherently random due to the unpredictability of person-to-person contacts [9]. Therefore, to understand the effect of stochastic factor in the influenza spread, we introduce stochastic noise into model parameter. Since the environmental stochasticity factor is related to the successful contact rate $\beta, \beta d t$ is then changed into a random variable $\tilde{\beta} d t$, that is

$$
\tilde{\beta} d t=\beta d t+\sigma d W(t)
$$

where $\sigma$ is a positive constant that denotes the noise intensity and $d W(t)=$ $W(t+d t)-W(t)$ is the increment of Wiener process.

Replacing $\beta d t$ with $\tilde{\beta} d t$, the deterministic model in [6] becomes the Itô SDE as follows

$$
\begin{aligned}
d S(t)= & \left(-\frac{\beta}{N} S\left(u_{1}[p-1]+1\right) I+\left(\alpha+\gamma_{0}+\gamma_{1} u_{2}\right) I\right) d t \\
& -\sigma \frac{S}{N} I\left(u_{1}[p-1]+1\right) d W(t) \\
d I(t)= & \left(\frac{\beta}{N} S\left(u_{1}[p-1]+1\right) I-\left(\alpha+\gamma_{0}+\gamma_{1} u_{2}\right) I\right) d t \\
& +\sigma \frac{S}{N} I\left(u_{1}[p-1]+1\right) d W(t) .
\end{aligned}
$$

Since the population is assumed to be constant $(S(t)+I(t)=N)$, it is sufficient to study the characteristics of solution of SDE (3b) only. 


\subsection{The SDE Model Solution}

The deterministic model solution and basic reproduction number $\left(R_{0}\right)$ were already discussed in [6]. The basic reproduction number $\left(R_{0}\right)$ of the deterministic model [6] as follows

$$
R_{0}=\frac{\beta\left(u_{1}[p-1]+1\right)}{\left(\alpha+\gamma_{0}+\gamma_{1} u_{2}\right)},
$$

and $\lim _{t \rightarrow \infty} I(t)=0$ if and only if $R_{0} \leq 1$.

Let

$$
\rho_{0}=\frac{\beta\left(u_{1}[p-1]+1\right)}{\left(\alpha+\gamma_{0}+\gamma_{1} u_{2}\right)}-\frac{\sigma^{2}\left(u_{1}[p-1]+1\right)^{2}}{2\left(\alpha+\gamma_{0}+\gamma_{1} u_{2}\right)},
$$

as the stochastic threshold parameter of SDE (3b) and the value of $\rho_{0}$ can be negative. The following theorem discuss the characteristic of $\rho_{0}$ related to the extinction and persistence condition of influenza spread.

Theorem 1. If

$$
\rho_{0}=\frac{\beta\left(u_{1}[p-1]+1\right)}{\left(\alpha+\gamma_{0}+\gamma_{1} u_{2}\right)}-\frac{\sigma^{2}\left(u_{1}[p-1]+1\right)^{2}}{2\left(\alpha+\gamma_{0}+\gamma_{1} u_{2}\right)}<1,
$$

and

$$
\sigma^{2}\left(u_{1}[p-1]+1\right)^{2} \leq \beta\left(u_{1}[p-1]+1\right)
$$

then for any initial value $I(0)=I_{0} \in(0, N)$, the solution of $S D E$ (3b) obeys

$$
\begin{aligned}
& \limsup _{t \rightarrow \infty} \frac{1}{t} \log I(t) \leq \beta\left(u_{1}[p-1]+1\right)-\left(\alpha+\gamma_{0}+\gamma_{1} u_{2}\right)- \\
& \frac{1}{2}\left(\sigma\left(u_{1}[p-1]+1\right)\right)^{2}<0 \quad \text { a.s. }
\end{aligned}
$$

namely, $I(t)$ tends to zero exponentially almost surely, i.e. the disease dies out with probability one.

Proof. Write $d I(t)$ in $(3 \mathrm{~b})$ as

$$
\begin{aligned}
d I(t)= & \left(\beta I\left(u_{1}[p-1]+1\right)-\frac{\beta}{N} I^{2}\left(u_{1}[p-1]+1\right)-\left(\alpha+\gamma_{0}+\gamma_{1} u_{2}\right) I\right) d t \\
& +\sigma \frac{N-I}{N} I\left(u_{1}[p-1]+1\right) d W(t) .
\end{aligned}
$$


To prove implication (8), use Itô formula by letting $Y(t)=\log I(t)$, hence from (9) we get

$$
\log I(t)=\log I_{0}+\int_{0}^{t} f(I(s)) d s+\int_{0}^{t} \frac{\sigma}{N}(N-I)\left(u_{1}[p-1]+1\right) d W(s),
$$

where $f: \mathbb{R} \rightarrow \mathbb{R}$ is defined by

$$
\begin{aligned}
f(I)= & \beta\left(u_{1}[p-1]+1\right)-\frac{\beta}{N} I\left(u_{1}[p-1]+1\right)-\left(\alpha+\gamma_{0}+\gamma_{1} u_{2}\right)- \\
& \frac{1}{2}\left(\sigma\left(1-\frac{I}{N}\right)\left(u_{1}[p-1]+1\right)\right)^{2}
\end{aligned}
$$

Under condition (7), we have

$$
\begin{aligned}
\log I(t) \leq & \log I(0)+t\left(\beta\left(u_{1}[p-1]+1\right)-\left(\alpha+\gamma_{0}+\gamma_{1} u_{2}\right)\right. \\
& \left.-\frac{1}{2} \sigma^{2}\left(u_{1}[p-1]+1\right)^{2}\right)+\int_{0}^{t} \sigma \frac{N-I}{N}\left(u_{1}[p-1]+1\right) d W(s) .
\end{aligned}
$$

This implies

$$
\begin{aligned}
& \limsup _{t \rightarrow \infty} \frac{1}{t} \log I(t) \leq\left(\beta\left(u_{1}[p-1]+1\right)-\left(\alpha+\gamma_{0}+\gamma_{1} u_{2}\right)\right. \\
& \left.-\frac{1}{2} \sigma^{2}\left(u_{1}[p-1]+1\right)^{2}\right)+\limsup _{t \rightarrow \infty} \frac{1}{t} \int_{0}^{t} \sigma \frac{N-I}{N}\left(u_{1}[p-1]+1\right) d W(s)
\end{aligned}
$$

Based on [8],

$$
\lim _{t \rightarrow \infty} \frac{1}{t} W(t)=0 \quad \text { a.s. }
$$

Hence, equation (12) becomes

$$
\begin{aligned}
& \limsup _{t \rightarrow \infty} \frac{1}{t} \log I(t) \leq \beta\left(u_{1}[p-1]+1\right)-\left(\alpha+\gamma_{0}+\gamma_{1} u_{2}\right)- \\
& \frac{1}{2} \sigma^{2}\left(u_{1}[p-1]+1\right)^{2} \text { a.s. }
\end{aligned}
$$

Under condition (6), it then follows from (14) that

$$
\begin{aligned}
\limsup _{t \rightarrow \infty} \frac{1}{t} \log I(t) \leq & \beta\left(u_{1}[p-1]+1\right)-\left(\alpha+\gamma_{0}+\gamma_{1} u_{2}\right)- \\
& \frac{1}{2}\left(\sigma\left(u_{1}[p-1]+1\right)\right)^{2}<0 \quad \text { a.s. }
\end{aligned}
$$


Compared to deterministic reproduction number which reach the extinction of Influenza if and only if $R_{0} \leq 1, \rho_{0}$ in Theorem 1 needs an additional term, namely $\sigma^{2}\left(u_{1}[p-1]+1\right)^{2} \leq \beta\left(u_{1}[p-1]+1\right)$. However, by listing all possible conditions that make $\rho_{0} \leq 1$, we can prove the extinction of disease which is analogous with $R_{0}$ of deterministic model. Such conditions are discussed below.

Theorem 2. If

$$
\sigma^{2}\left(u_{1}[p-1]+1\right)^{2}>\max \left(\beta\left(u_{1}[p-1]+1\right), \frac{\beta^{2}\left(u_{1}[p-1]+1\right)^{2}}{2\left(\alpha+\gamma_{0}+\gamma_{1} u_{2}\right)}\right),
$$

then the value of stochastic threshold parameter $\rho_{0} \leq 1$ and for any initial value $I(0)=I_{0} \in(0, N)$, the solution of SDE (3b) obeys

$$
\limsup _{t \rightarrow \infty} \frac{1}{t} \log I(t) \leq \frac{1}{2} \frac{\beta^{2}}{\sigma^{2}}-\left(\alpha+\gamma_{0}+\gamma_{1} u_{2}\right)<0 \quad \text { a.s. }
$$

namely, $I(t)$ tends to zero exponentially almost surely, i.e. the disease dies out with probability one.

Proof. Consider condition (16) into two separate cases, that is Case 1.

$$
\sigma^{2}\left(u_{1}[p-1]+1\right)^{2}>\beta\left(u_{1}[p-1]+1\right),
$$

which implies

$$
\beta\left(u_{1}[p-1]+1\right) \geq \frac{\beta^{2}\left(u_{1}[p-1]+1\right)^{2}}{2\left(\alpha+\gamma_{0}+\gamma_{1} u_{2}\right)} .
$$

The equation (18) can be written as

$$
\frac{\beta\left(u_{1}[p-1]+1\right)}{2\left(\alpha+\gamma_{0}+\gamma_{1} u_{2}\right.} \leq 1 .
$$

By considering case 1 in (18), $\rho_{0}$ in equation (5) can be written as

$$
\rho_{0} \leq \frac{\beta\left(u_{1}[p-1]+1\right)}{\left(\alpha+\gamma_{0}+\gamma_{1} u_{2}\right)}-\frac{\beta\left(u_{1}[p-1]+1\right)}{2\left(\alpha+\gamma_{0}+\gamma_{1} u_{2}\right)}=\frac{\beta\left(u_{1}[p-1]+1\right)}{2\left(\alpha+\gamma_{0}+\gamma_{1} u_{2}\right)},
$$

and by using equation (20), the last equation becomes

$$
\rho_{0} \leq \frac{\beta\left(u_{1}[p-1]+1\right)}{2\left(\alpha+\gamma_{0}+\gamma_{1} u_{2}\right)} \leq 1
$$


Case 2 .

$$
\sigma^{2}\left(u_{1}[p-1]+1\right)^{2}>\frac{\beta^{2}\left(u_{1}[p-1]+1\right)^{2}}{2\left(\alpha+\gamma_{0}+\gamma_{1} u_{2}\right)}
$$

which implies

$$
\frac{\beta^{2}\left(u_{1}[p-1]+1\right)^{2}}{2\left(\alpha+\gamma_{0}+\gamma_{1} u_{2}\right)} \geq \beta\left(u_{1}[p-1]+1\right)
$$

The proof is analogue, thus both cases show that $\rho_{0} \leq 1$. Furthermore, summoning the function (11) and equation (18), it takes its maximum value $f\left(I_{*}\right)$ at the stationary point

$$
I_{*}=N\left(1-\frac{\beta}{\sigma^{2}\left(u_{1}[p-1]+1\right)}\right) \in(0, N) .
$$

From equation (22), the maximum value of function (11) is

$$
f\left(I_{*}\right)=\frac{1}{2} \frac{\beta^{2}}{\sigma^{2}}-\left(\alpha+\gamma_{0}+\gamma_{1} u_{2}\right)<0 .
$$

Recall the log function (10) and use equation (25) together with equation (13) implies

$$
\limsup _{t \rightarrow \infty} \frac{1}{t} \log I(t) \leq \frac{1}{2} \frac{\beta^{2}}{\sigma^{2}}-\left(\alpha+\gamma_{0}+\gamma_{1} u_{2}\right)<0 \quad \text { a.s. }
$$

Now the deterministic and stochastic threshold parameter are analogous, that is the disease vanishes when the threshold parameter value is less or equal to one. The following theorem discusses the persistence of Influenza in population.

Theorem 3. If $\rho_{0}>1$, then for any initial value $I(0)=I_{0} \in(0, N)$, the solution of SDE (3b) obeys

$$
\liminf _{t \rightarrow \infty} I(t) \leq I_{\xi} \leq \limsup _{t \rightarrow \infty} I(t) \quad \text { a.s. }
$$

where

$$
I_{\xi}=\frac{N\left(\sqrt{\beta^{2}-2 \sigma^{2}\left(\alpha+\gamma_{0}+\gamma_{1} u_{2}\right)}-\left(\beta-\sigma^{2}(u 1[p-1]+1)\right)\right)}{\sigma^{2}\left(u_{1}[p-1]+1\right)} .
$$


Proof. Before proving further, we bring forth $I_{\xi}$ in equation (27) first by considering the function $f(I)$. Note that $f(I)$ in equation (11) have positive and negative root and we only consider the positive root. Under condition that $\rho_{0}>$ 1 , it is found that $I_{\xi}$ is the unique positive root of $f(I)$ and $f(0)>0$. In addition, due to the positive values of $\alpha, \gamma_{0}, \gamma_{1}$ and $u_{2}, f(N)=-\left(\alpha+\gamma_{0}+\gamma_{1} u_{2}\right)<0$. Furthermore, by comparing $I_{\xi}$ to the stationary point $I_{*}$, it is easy to see that $I_{*}<I_{\xi}$. which makes $f\left(I_{*}\right)$ possibly the candidate of maximum value of $f(I)$ at

$$
f\left(I_{*}\right)=\frac{\beta^{2}}{2 \sigma^{2}}-\left(\alpha+\gamma_{0}+\gamma_{1} u_{2}\right)>0 .
$$

It follows that if $f\left(I_{*}\right) \geq f(0)$,

$$
\begin{gathered}
f(I)>0 \quad \text { increasing function on } I \in\left(0, I_{*}\right), \\
f(I)>0 \text { strictly decreasing function on } I \in\left(I_{*}, I_{\xi}\right),
\end{gathered}
$$

and

$$
f(I)<0 \quad \text { strictly decreasing function on } I \in\left(I_{\xi}, N\right) .
$$

Otherwise if $f(0) \geq f\left(I_{*}\right)$,

$$
f(I)>0 \text { decreasing function on } I \in\left(0, I_{\xi}\right),
$$

and

$$
f(I)<0 \quad \text { strictly decreasing function on } I \in\left(I_{\xi}, N\right) .
$$

To show assertion (26), first we prove that

$$
\liminf _{t \rightarrow \infty} I(t) \leq I_{\xi}
$$

is true. Suppose that (34) is not true, that is $\liminf _{t \rightarrow \infty} I(t)>I_{\xi} \quad$ a.s., then there is $\delta \in(0,1)$ such that $\mathbb{P}\left(\Omega_{1}\right)>\delta$ where $\Omega_{1}=\left\{\liminf _{t \rightarrow \infty} I(t) \geq I_{\xi}+2 \delta\right\}$. That is, for all $\omega \in \Omega_{1}$ there is $t=\tau>0$ such that

$$
I(t) \geq I_{\xi}+\delta \quad \text { as } \quad t \geq \tau
$$

From (31), (33), and (35), we can say

$$
f\left(I(t) \leq f\left(I_{\xi}+\delta\right)<f\left(I_{\xi}\right)=0 \quad \text { as } \quad t \geq \tau .\right.
$$

Recalling (10), it then follows that

$$
\log I(t) \leq \log I(0)+\int_{0}^{\tau} f(I(s)) d s+f\left(I_{\xi}+\delta\right)(t-\tau)+\int_{0}^{t} \sigma \frac{(N-I)}{N}\left(u_{1}[p-1]+1\right) d W(t) .
$$


Using equation (13) and (36), equation (37) becomes

$$
\limsup _{t \rightarrow \infty} \frac{1}{t} \log I(t) \leq f\left(I_{\xi}+\delta\right)<0 \quad \text { a.s. }
$$

Based on [8], inequality (38) implies that

$$
\lim _{t \rightarrow \infty} I(t)=0
$$

This contradicts (35). Therefore, (34) must be true. Furthermore, to prove that $I_{\xi} \leq \limsup _{t \rightarrow \infty} I(t)$ a.s., we go through similar manner.

Note that level of $I_{\xi}$ is the endemic level for $I(t)$ in stochastic model. By definition of $R_{0}$ in (4), $\rho_{0}$ now can be written as follows.

$$
\rho_{0}=\frac{\beta\left(u_{1}[p-1]+1\right)}{\left(\alpha+\gamma_{0}+\gamma_{1} u_{2}\right)}-\frac{\sigma^{2}\left(u_{1}[p-1]+1\right)^{2}}{2\left(\alpha+\gamma_{0}+\gamma_{1} u_{2}\right)}=R_{0}-\frac{\sigma^{2}\left(u_{1}[p-1]+1\right)^{2}}{2\left(\alpha+\gamma_{0}+\gamma_{1} u_{2}\right)} \text {. }
$$

If $R_{0}>1$, there exist certain value of $\sigma$ such that $\rho_{0}<1$. It means that while deterministic model indicates persistence of a disease, the stochastic model may indicate the extinction of a disease, which also stated in [5].

\section{Results}

\subsection{Sensitivity Analysis of $\rho_{0}$}

The sensitivity analysis is presented to show the change of $\rho_{0}$ value when some parameters are changed, while the rest held constant. In this session, we observe how the change of parameter $u_{1}$ and $u_{2}$ affect the change of $\rho_{0}$ value in Figure 2 (for two cases, let call it as small $(\sigma=0.05)$ and large $(\sigma=0.15)$ stochastic environmental factor ). The constant parameters and their description is given in Table 1 (except it is stated otherwise).

In Figure 2, each coloured line presents the magnitude of $\rho_{0}$ with respect to $u_{1}$ and $u_{2}$ values. Even though both figures show similar characteristic that is enlarging $u_{1}$ and $u_{2}$ will reduce $\rho_{0}$, note that between Figure $2(\mathrm{a})$ and $(\mathrm{b})$, there is a significant shift in $\rho_{0}$ values. Suppose $\sigma$ is small enough $(\sigma=0.05)$ in (a), if nobody gets drug treatment $\left(u_{2}=0\right)$, then in order to become extinct, that is $\rho_{0} \leq 1$, we need more than $30 \%$ infected individuals to wear medical mask. Whereas with bigger value of $\sigma$ in (b), we only need at least $22 \%$ infected individuals to wear medical mask so that the disease dies out.

Next sensitivity analysis in Figure 3 is given to compare the value of $u_{1}$ and $u_{2}$ with respect to $R_{0}$ and $\rho_{0}$. Figure 3 consists of three different region : 


\begin{tabular}{|c|c|l|}
\hline Parameters & Values & Description \\
\hline$N$ & 100 & We assume total population is 100 people \\
\hline$\beta$ & $0.1 \frac{\text { person }}{\text { day }}$ & $\begin{array}{l}\text { It needs } 10 \text { successful contacts with in- } \\
\text { fected person to transmit influenza }\end{array}$ \\
\hline$\alpha$ & $\frac{1}{71 \times 365 \text { days }}$ & Based on WHO life expectancy [23] \\
\hline$\gamma_{0}$ & $\frac{1}{14 \text { days }}$ & $\begin{array}{l}\text { We assume the natural recovery rate for } \\
\text { influenza disease is } 14 \text { days }\end{array}$ \\
\hline$\gamma_{1}$ & $\frac{1}{14 \text { days }}$ & $\begin{array}{l}\text { We assume that drug treatment will in- } \\
\text { crease recovery rate by a half from } \frac{1}{14} \text { to } \\
\frac{1}{7} \text {. Therefore, we choose } \gamma_{1}=\frac{1}{14} \text {. Please } \\
\text { see details in [6]. }\end{array}$ \\
\hline$p$ & 0.1 & $\begin{array}{l}\text { We assume that medical mask will reduce } \\
\text { the risk of getting influenza by 90\%. }\end{array}$ \\
\hline$u_{1}$ & $u_{1} \in[0,1]$ & The rate of used medical mask each day \\
\hline$u_{2}$ & $u_{2} \in[0,1]$ & $\begin{array}{l}\text { The rate of drug treatment intervention } \\
\text { each day }\end{array}$ \\
\hline
\end{tabular}

Table 1: Parameters description

1. Region 1 . In this region, both deterministic and stochastic model present the same result i.e disease will dies out. It is shown by the magnitude of $R_{0}$ and $\rho_{0}$ are equal or smaller than one.

2. Region 2 . In this region, a contradiction result between deterministic and stochastic model is obtained, where $R_{0}>1$ but in the other hand $\rho_{0}<1$. This result presents that the stochastic environment has an important role in determining the existence of disease.

3. Region 3 . In this region, both deterministic and stochastic model present the same result i.e disease will exist. It is shown by the magnitude of $R_{0}$ and $\rho_{0}$ are larger than one.

As a consequence of the environment perturbation, we can find $\rho_{0}<1$ but in the other hand $R_{0}>1$ with the same used parameter values. In other words, we can find some situations when deterministic and SDE model will gave a different result about the coexistence of influenza disease depend on how large is the stochastic environment factor. 


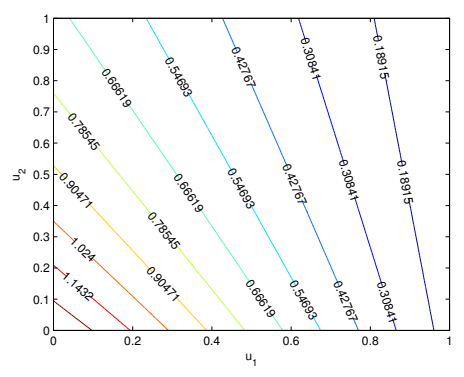

(a)

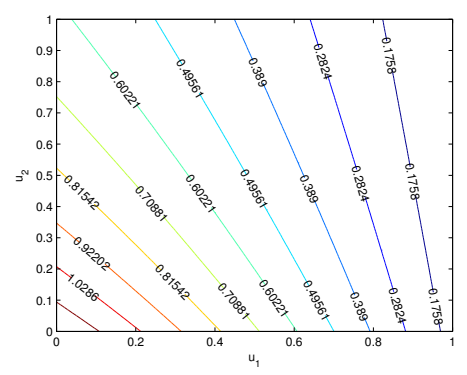

(b)

Figure 2: Sensitivity analysis of $\rho_{0}$ with respect to $u_{1}$ and $u_{2}$ for (a) $\sigma=0.05$ and (b) $\sigma=0.15$

\subsection{Numerical Simulations}

In this subsection, some numerical simulations are shown to give an interpretation about analytical results discussed in previous section. There are some cases to discuss as follows.

Simulating the deterministic model (1), we discuss the prevention and reduction of an Influenza outbreak that is shown in Figure 5. We then separate the case where an outbreak just happen, that is the initial number of infected person is above the endemic level (see Figure 5 (a) in which we analyse the endemic reduction plan using medical mask and drug), and another case where an outbreak does not happen yet that is depicted in Figure 5 (b) which shows the endemic prevention using the same method.

We found that in the Figure 5 (a), medical mask can reduce the disease up to $86 \%$ of the infected population, while drug treatment can only bring down $74 \%$ of it. Figure 5 (b) releases similar result, that is, medical mask can reduce the disease up to $98 \%$ of the infected population, while drug treatment can only bring down $84 \%$. From both figure above, we see that preventing and reducing the disease using medical mask is more effective than treatment with drugs.

Next, we simulate the stochastic model (3b). The first simulation is performed to show an initial situation when there are no interventions given to the population $\left(u_{1}=u_{2}=0\right)$. We assume that $R_{0}>1$ and $\rho_{0}>1$ in $t=0$. For this purpose, parameters value are given in table 1 . It is important to stress that the mortality and recovery parameter values $\left(\alpha, \gamma_{0}\right.$ and $\left.\gamma_{1}\right)$ remain the same, while the infection rate $\beta$ and the intervention parameter values $\left(u_{1}\right.$ and $\left.u_{2}\right)$ 


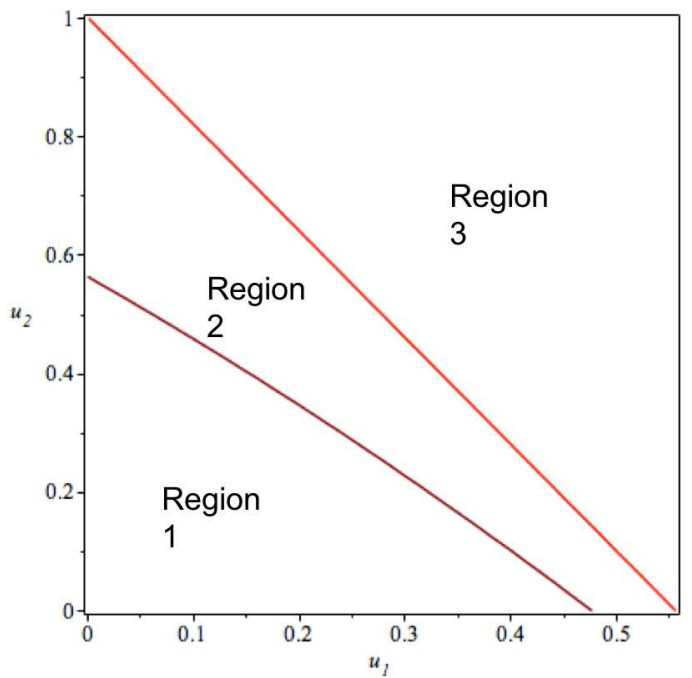

Figure 3: Comparison of sensitivity between $u_{1}$ and $u_{2}$ for $R_{0}$ and $\rho_{0}$.

are changed in order to explore the behaviour of the Influenza spread.

Figure 6 generally show that the trend of the stochastic solutions agree with the corresponding deterministic solutions. Note that the number of infected individuals starts small and continuously rise up to an endemic level. As a consequence, the number of susceptible individuals becomes less than its initial value as $t$ increases. Observe that Figure 6 (right figure) suggests that as the infection rate is higher, sat a certain time $t$ the number of infected individuals is larger than the susceptible.

Second, the case where $R_{0}<1$ and $\rho_{0}<1$. This case considers the intervention of medical mask and drug treatment to a half of population, that is $u_{1}=u_{2}=0.5$. By using interventions, some significant changes from the first simulation are served in Figure 7. The Influenza disease dies out starting from certain time and the whole population become susceptible. To investigate the effect of varying the infection rate on our model, $\beta$ was given different sets of values. We see that the disease disappears sooner with lower rate of infection. Lastly, the case where $R_{0}>1$ and $\rho_{0}<1$. Using parameter value $\beta=0.143$, Figure 8 depict the contradictory situation between deterministic and stochastic solutions. Figure 8 (a), where $R_{0}=1.01$ and $\rho_{0}=0.99$, the deterministic graph shows that in the long term the infected individuals do not go away from the population at endemic equilibrium $I_{\xi}=1.8$ while the stochastic solution 


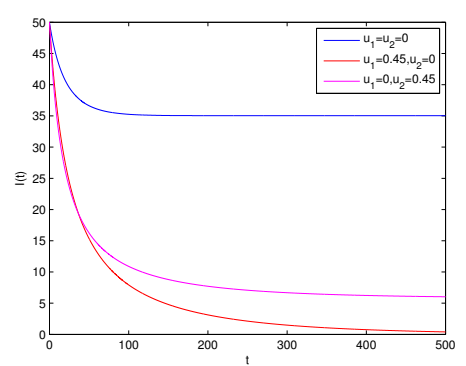

(a)



(b)

Figure 5: Endemic reduction and prevention
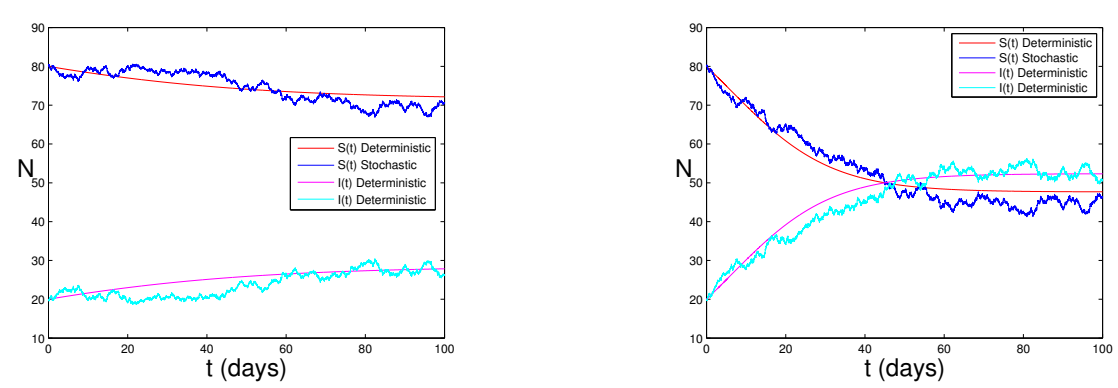

Figure 6: Stochastic and deterministic solutions for persistence condition with $\beta=0.1$ (left) and $\beta=0.15$ (right) .

show that the disease will eventually vanish. When compared to Figure 8 (b), which has a greater value of $R_{0}$ but a smaller $\rho_{0}\left(R_{0}=1.24, \rho_{0}=0.94\right)$, shows the disease will persist at a bigger endemic level $I_{\xi}=19$ by deterministic solution while the stochastic solution demonstrates a much sooner extinction of disease than Figure 8 (a).

\section{Conclusions}

In this paper, we have constructed a stochastic model for Influenza spread with medical mask $u_{1}$ and treatment $u_{2}$ intervention. From analysis of stochastic threshold parameter $\left(\rho_{0}\right)$, we find that enlarging $u_{1}$ and $u_{2}$ will reduce $\rho_{0}$. The disease will extinct if $\rho_{0}<1$ and persist otherwise. The disease will extinct sooner if the value of $\rho_{0}$ smaller. We also find that while deterministic model indicates persistence of a disease, the stochastic model may indicate the 

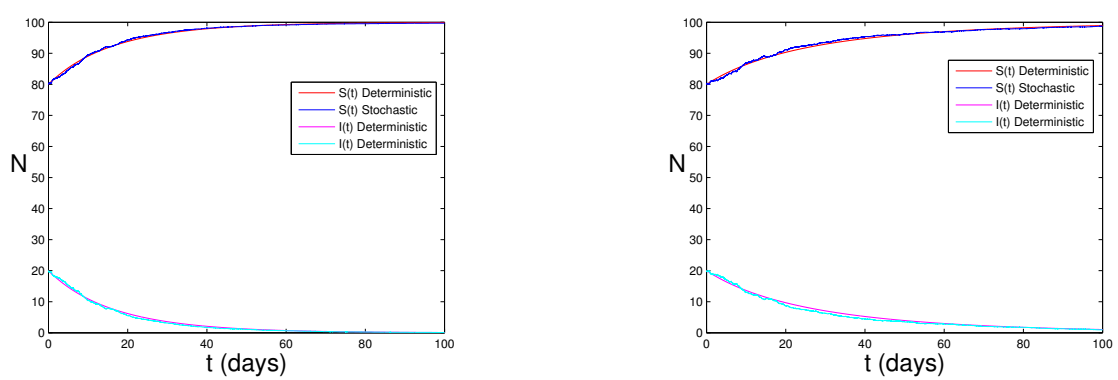

Figure 7: Stochastic and deterministic solution with (a) $\beta=0.1$ (b) $\beta=0.15$
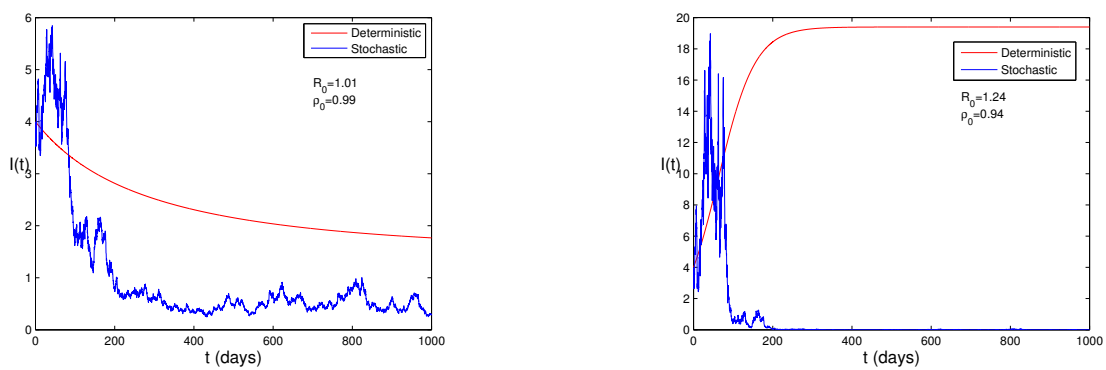

Figure 8: Stochastic and deterministic solution with (a) $\sigma=0.1, u_{1}=$ $u_{2}=0.35$ (b) $\sigma=0.3, u_{1}=u_{2}=0.25$

extinction of a disease.

\section{Acknowledgments}

The authors thanks to all reviewer for their constructed comments and suggestions. This research is funded by KEMRISTEK DIKTI research grant, under PUPT 2017 scheme.

\section{References}

[1] D.H. Ackley, G.E. Hinton and T.J. Sejnowski, A learning algorithm for Boltzmann machine, Cognitive Science, 9, (1985), 147-169

[2] F.L. Crane, H. Low, P. Navas, I.L. Sun, Control of cell growth by plasma membrane NADH oxidation, Pure and Applied Chemical Sciences, 1 (2013), 31 - 42. http://dx.doi.org/10.12988/pacs.2013.3310 
[3] D.O. Hebb, The Organization of Behavior, Wiley, New York, USA (1949).

[4] Center for Disease Control and Prevention, USA. Epidemiology and Prevention of Vaccine-Preventable Diseases : Influenza. October 5, 2015.

[5] Herwaarden, O. A. and Grasman, J, . Stochastic epidemics: major outbreaks and the duration of the endemic period. Journal of Mathematical Biology. 33, No. 6 (1995), 581601.

[6] F. Novkaniza, Ivana, D.Aldila, Comparison Between Discrete Time Markov Chain and Deterministic Model, AIP Conf. Proc. 1723 (2016), 1-9. doi: 10.1063/1.4945073 .

[7] Kermack, W.O. and McKendrick, A.G., Contributions to the mathematical theory of epidemics-Part I. Proceedings of the Royal Society Series A, 115 (1927), 700-721.

[8] Mao, X. . Stochastic differential equations and applications. (2nd ed.). Chichester: Horwood Publishing Limited. (2007).

[9] Spencer, S. . Stochastic epidemic models for emerging diseases. PhD thesis. University of Nottingham. (2008).

[10] WHO. . Cumulative number of confirmed human cases of Avian Influenza A (H5N1) reported to WHO. http://www.who.int. (2006).

[11] G.A.Ngwa, W.S.Shu, A mathematical model for endemic malaria with variable human and mosquito populations, Mathematical and Computer Modelling, 32 (2000), Issues 7-8, 747-763.

[12] F. Forouzannia, A.B. Gumel, Mathematical analysis of an age-structured model for malaria transmission dynamics, Mathematical Biosciences, 247 (2014), 80-94.

[13] C. Bowman, A.B. Gumel, P. van den Driessche, J. Wu, H. Zhu, A mathematical model for assessing control strategies against West Nile virus, Bulletin of Mathematical Biology, 67 (2005), Issue 5, 1107-1133 .

[14] S.M.Garba, M.A.Safi, Mathematical Analysis of West Nile Virus Model with Discrete Delays, Acta Mathematica Scientia, 33 (2013), Issue 5, 1439-1462 .

[15] D.Aldila, T.Goetz, E.Soewono, An optimal control problem arising from a dengue disease transmission model, Mathematical Biosciences, 242 (2013), Issue 1, 9-16 .

[16] D Aldila, N Nuraini, E Soewono, AK Supriatna, Mathematical model of temephos resistance in Aedes aegypti mosquito population, AIP Conf. Proc. 1589 (2014), Issue 1, doi : 10.1063/1.4868843.

[17] D. Aldila, N.Nuraini. E.Soewono, Optimal Control Problem in Preventing of Swine Flu Disease Transmission, Applied Mathematical Sciences, 8 (2014), no. 71, 3501 - 3512.

[18] Meksianis Z. Ndii, R.I. Hickson, David Allingham, G.N. Mercer, Modelling the transmission dynamics of dengue in the presence of Wolbachia, Mathematical Biosciences, 262 (2015), 157-166.

[19] S. Iwami, Y. Takeuchi, X. Liu, Avianhuman influenza epidemic model, Mathematical Biosciences, 207 (2007), Issue 1, 1-25.

[20] T. C. Germann, K. Kadau, I. M. Longini, Jr., C. A. Macken, Mitigation strategies for pandemic influenza in the United States, PNAS, 103 (2006), issue 15, 5935-5940 .

[21] C.E. Mills, J.M. Robins, C.T. Bergstrom, M. Lipsitch, Pandemic in- fluenza: risk of multiple introductions and the need to prepare for them, PLoS Medicine, 3 (2006), issue $6,1-5$. 
[22] A. Le Menach, E. Vargu, R. F. Grais, D. L. Smith, A. Flahault, Key strategies for reducing spread of avian influenza among commercial poultry holdings: lessons for transmission to humans, Proc. R. Soc. B, 273 (2006), 2467-2475 .

[23] www.who.int, accessed in 31 October 2016. 